PROCEEDINGS OF THE

AMERICAN MATHEMATICAL SOCIETY

Volume 125, Number 2, February 1997, Pages 363-373

S 0002-9939(97)03602-2

\title{
A NOTE ON GK DIMENSION OF SKEW POLYNOMIAL EXTENSIONS
}

\author{
JAMES J. ZHANG
}

(Communicated by Ken Goodearl)

\begin{abstract}
Let $A$ be a finitely generated commutative domain over an algebraically closed field $k, \sigma$ an algebra endomorphism of $A$, and $\delta$ a $\sigma$-derivation of $A$. Then $\operatorname{GKdim}(A[x, \sigma, \delta])=\operatorname{GKdim}(A)+1$ if and only if $\sigma$ is locally algebraic in the sense that every finite dimensional subspace of $A$ is contained in a finite dimensional $\sigma$-stable subspace.

Similarly, if $F$ is a finitely generated field over $k, \sigma$ a $k$-endomorphism of $F$, and $\delta$ a $\sigma$-derivation of $F$, then $\operatorname{GKdim}(F[x, \sigma, \delta])=\operatorname{GKdim}(F)+1$ if and only if $\sigma$ is an automorphism of finite order.
\end{abstract}

\section{THEOREMS}

Throughout this paper, $k$ denotes an algebraically closed field and $A$ will be a $k$-algebra. Let $\sigma$ be a $k$-algebra endomorphism of $A$. Then the algebra $A[x, \sigma]$ is a polynomial extension of $A$ subject to the relation $a x=x \sigma(a)$ for all $a \in A$. If $\sigma$ is an automorphism, then $x$ is a regular normal elment in $A[x, \sigma]$ and the algebra $A\left[x, x^{-1}, \sigma\right]$ is defined by inverting $x$ in $A[x, \sigma]$. Let GKdim denote the Gelfand-Kirillov dimension. The definition and basic properties of GKdim can be found in [KL]. A finite dimensional subspace of $A$ containing the identity 1 is called a subframe of $A$. A subframe (or a subspace) $V$ of $A$ is said to be $\sigma$-stable if $\sigma(V) \subset V$. We call $\sigma$ locally algebraic if every subframe of $A$ is contained in a $\sigma$ stable subframe. It is not hard to prove that if $\sigma$ is a locally algebraic automorphism, then $\operatorname{GKdim}\left(A\left[x, x^{-1}, \sigma\right]\right)=\operatorname{GKdim}(A)+1[$ LMO, Prop. 1]. In this paper we will prove a partial converse when $A$ is a commutative domain.

Theorem 1.1. Let $A$ be a commutative domain over $k$ such that the fraction field $Q(A)$ is finitely generated as a field, and let $\sigma$ be an endomorphism of $A$. Then the following statements are equivalent.

(a) $\operatorname{GKdim}(A[x, \sigma])<\operatorname{GKdim}(A)+2$.

(a) $\operatorname{GKdim}\left(A\left[x, x^{-1}, \sigma\right]\right)<\operatorname{GKdim}(A)+2$ if $\sigma$ is an automorphism.

(b) $\operatorname{GKdim}(A[x, \sigma])=\operatorname{GKdim}(A)+1$.

(b) $\operatorname{GKdim}\left(A\left[x, x^{-1}, \sigma\right]\right)=\operatorname{GKdim}(A)+1$ if $\sigma$ is an automorphism.

(c) $\sigma$ is locally algebraic.

Received by the editors June 19, 1995 and, in revised form, August 24, 1995.

1991 Mathematics Subject Classification. Primary 16P90, 16 S36.

Key words and phrases. Gelfand-Kirillov dimension, Polynomial extension, automorphism of algebra.

This research was supported in part by the NSF. 
If moreover $A$ is a field, then (a)-(c) are equivalent to the following.

(d) $\sigma$ is an automorphism of finite order.

The only difficult step is the implication from (a) to (c). This implication will fail if either (i) $A$ is not a domain or (ii) $A$ is not commutative (see Examples in $\S 5$ ). A related example was given by I. Musson $[\mathrm{Mu}]$ which shows that there is a non-prime PI algebra $A$ and an algebra automorphism $\sigma$ of $A$ such that there are no non-trivial $\sigma$-stable subspaces of $A$ and that $\operatorname{GKdim}\left(A\left[x, x^{-1}, \sigma\right]\right)=\operatorname{GKdim}(A)+1=3$. It is unknown if Theorem 1.1 holds for prime PI algebras.

Let $\delta$ be a $\sigma$-derivation of $A$, i.e., $\delta(a b)=\delta(a) \sigma(b)+a \delta(b)$ for every $a, b \in A$. Then the algebra $A[x, \sigma, \delta]$ is a polynomial extension of $A$ satisfying the relation $a x=x \sigma(a)+\delta(a)$ for all $a \in A$ (see [MR, Sec. 1.2]). A subspace $V$ of $A$ is called $\delta$-stable if $\delta(V) \subset V$. We will prove the following analogue to Theorem 1.1.

Theorem 1.2. Let $A$ be a commutative domain over $k, \sigma$ an algebra endomorphism of $A$, and $\delta$ a $\sigma$-derivation of $A$.

(1) If $A$ is a finitely generated algebra, then the following statements are equivalent:

(a) $\operatorname{GKdim}(A[x, \sigma, \delta])<\operatorname{GKdim}(A)+2$.

(b) $\operatorname{GKdim}(A[x, \sigma, \delta])=\operatorname{GKdim}(A)+1$.

(c) $\sigma$ is locally algebraic.

(2) If $A$ is a finitely generated field, then each of (a)-(c) is equivalent to the following:

(d) $\sigma$ is an automorphism of finite order.

In $\S 2$ we will prove a lemma which will be used several times in later sections. Section 3 is devoted to the proof of Theorem 1.1, and $\S 4$ contains the proof of Theorem 1.2. In $\S 5$ we will give some examples which show that the hypotheses in Theorems 1.1 and 1.2 are necessary.

\section{A LEMMA}

The lemma we will prove is essentially contained in [Zh, Sec. 6]. Let $G$ be an ordered semigroup with unit $e$. Familiar examples of ordered (semi)groups are $\mathbb{N}^{l}$ and $\mathbb{Z}^{l}$ for $l \geq 1$. Let $A$ be an algebra with a filtration $\left\{F_{n} \mid n \in G\right\}$ of subspaces of $A$. We denote by $F_{<n}$ the subspace $\sum_{m<n} F_{m}$. Suppose the filtration satisfies the following conditions:

(f1) $F_{m} \subset F_{n}$ for all $m<n$ in $G$;

(f2) $F_{m} F_{n} \subset F_{m n}$ for all $m$ and $n$ in $G$;

(f3) $A=\bigcup_{n \in G}\left(F_{n}-F_{<n}\right)$;

(f4) $1 \in F_{e}-F_{<e}$.

Then $A$ is called a $G$-filtered algebra. The associated graded algebra is defined to be $\operatorname{gr}(A)=\bigoplus_{n \in G} F_{n} / F_{<n}$ with the $k$-linear multiplication determined by

$$
\left(a+F_{<m}\right)\left(b+F_{<n}\right)=a b+F_{<n m}
$$

(see [KL, page 73] for the case when $G=\mathbb{Z}$ ). We define a map $\nu: A \longrightarrow \operatorname{gr}(A)$ by $\nu(a)=a+F_{<n}$ for all $a \in F_{n}-F_{<n}$. This map is called a leading-term map and it is easy to see that

(11) $\nu(t)=t$ for all $t \in k$;

(12) $\nu(a) \neq 0$ for all $a \neq 0$; 
(13) $\nu(a) \nu(b)=\nu(a b)$ or $\nu(a) \nu(b)=0$ for all $a, b \in A$.

If the associated graded algebra $\operatorname{gr}(A)$ is a domain, then $\nu(a) \nu(b)=\nu(a b)$ for all $a, b \in A$.

Obvious examples of filtered algebras are graded algebras. A $G$-graded algebra $A=\bigoplus_{i \in G} A_{i}$ can be viewed naturally as a filtered algebra by letting $F_{n}=$ $\bigoplus_{m \leq n} A_{m}$. Then $\left\{F_{n} \mid n \in G\right\}$ is a filtration and $\operatorname{gr}(A)=A$. The leading-term map is $\nu(\bar{a})=a_{n_{g}}$ if $a=a_{n_{1}}+a_{n_{2}}+\cdots+a_{n_{g}}$ with $0 \neq a_{n_{i}} \in A_{n_{i}}$ and $n_{1}<n_{2}<\cdots<n_{g}$.

Some $\mathbb{N}$-filtered algebras can be characterized in the following way. Let $A$ be an algebra generated by a subframe $V$. Then $\left\{F_{i}=V^{i} \mid i \geq 0\right\}$ (where $V^{0}=$ $k$ ) is an $\mathbb{N}$-filtration of $A$ and $(\mathrm{f} 1,2,3,4)$ hold. The associated graded algebra is $\operatorname{gr}(A):=\bigoplus_{i} V^{i} / V^{i-1}$ and the leading-term map from $A$ to $\operatorname{gr}(A)$ is defined by $\nu: a \longmapsto a+V^{i-1}$ for all $a \in V^{i}-V^{i-1}$. Familiar examples are the Weyl algebras and universal enveloping algebras of finite dimensional Lie algebras. In these two examples, the associated graded algebras are domains.

Another example of an $\mathbb{N}$-filtered algebra is $A[x, \sigma, \delta]$ where $\sigma$ is an endomorphism of $A$ and $\delta$ is a $\sigma$-derivation of $A$. Let $F_{n}=\left\{\sum_{i \leq n} x^{i} a_{i} \mid a_{i} \in A\right\}$. Then $\left\{F_{n}\right\}$ satisfies $(\mathrm{f} 1,2,3,4)$ and $\operatorname{gr}(A[x, \sigma, \delta]) \cong A[x, \sigma]$.

The following lemma lists some basic properties of the leading-term map. For every subspace $V$ of $A$, we define $\nu(V)=\sum_{x \in V} k \nu(x)$. Hence $\nu(V)$ is a graded subspace of $\operatorname{gr}(A)$.

Lemma 2.1. Let $A$ be a $G$-filtered algebra and let $\nu$ be the leading-term map from A to $\operatorname{gr}(A)$.

(1) $\nu(V a) \supset \nu(V) \nu(a)$ for all $a \in A$ and $V \subset A$.

(2) $\nu(V+W) \supset \nu(V)+\nu(W)$ for all $V, W \subset A$.

(3) $\nu(V W) \supset \nu(V) \nu(W)$ for all $V, W \subset A$.

(4) $\operatorname{dim}(W)=\operatorname{dim}(\nu(W))$ for all $V \subset A$ where $\operatorname{dim}$ is the dimension of a $k$-vector space.

(5) $\operatorname{dim}(V W) \geq \operatorname{dim}(\nu(V) \nu(W))$ for all $V, W \subset A$.

(6) $\operatorname{dim}\left(W^{n}\right) \geq \operatorname{dim}\left((\nu(W))^{n}\right)$ for all $V \subset A$ and all $n \geq 1$.

(7) $\operatorname{GKdim}(A) \geq \operatorname{GKdim}(\operatorname{gr}(A))$.

Proof. (1) $\nu(V a)=\sum_{v} k \nu(v a) \supset \sum_{v} k \nu(v) \nu(a)=\left(\sum_{v} k \nu(v)\right) \nu(a)=\nu(V) \nu(a)$.

(2) Since $V \subset V+W, \nu(V) \subset \nu(V+W)$ and it follows that

$$
\nu(V)+\nu(W) \subset \nu(V+W) .
$$

(3) Write $V W=\sum_{w} V w$, then (3) follows from (1) and (2).

(4) Let $W_{n}=W \cap F_{n}$ and $W_{<n}=W \cap F_{<n}$ for all $n \in G$. Then $W_{n}$ and $W_{<n}$ are subspaces of $W$ and by the definition of $\nu$ we see that $\nu(W)=\bigoplus_{n} W_{n} / W_{<n}$. Hence

$$
\operatorname{dim}(\nu(W))=\sum_{n}\left(\operatorname{dim}\left(W_{n}\right)-\operatorname{dim}\left(W_{<n}\right)\right)=\operatorname{dim} W .
$$

(5) is a consequence of (3) and (4).

(6) is a consequence of (5) by induction.

(7) Let $V$ be a subframe of $\operatorname{gr}(A)$. There is a subframe $W$ of $A$ such that $\nu(W) \supset V$. (Recall that $\nu(W)=\sum_{x \in W} k \nu(x)$.) Then the statement follows from (6) and the definition of GKdim. 


\section{Proof of Theorem 1.1}

We divide the proof of Theorem 1.1 into two parts: Theorem 3.2 and Proposition 3.3 .

Let $A$ be a commutative algebra and let $V$ be a subframe of $A$. Applying [Ma, 13.2] to the graded algebra $B=\bigoplus_{n>0} V^{n} / V^{n-1}, \operatorname{dim}\left(V^{n}\right)$ is a polynomial of $n$ with rational coefficients for $n \gg 0$. If $\bar{A}$ is a commutative domain, we will show that the leading coefficient of the polynomial $\operatorname{dim}\left(V^{n}\right)$ is "at least linearly dependent on $\operatorname{dim}(V)$ " (the precise definition will be given below). Since the leading coefficient of the growth polynomial is related to the multiplicity [MR, 8.4.7], we make the following definition. Let $A$ be an algebra of GKdim $d$. We say $A$ satisfies the sensitive multiplicity condition (or SMC) if there is a finite dimensional subspace $V_{0}$ of $A$ and a constant $c>0$ such that if $W$ is a finite dimensional subspace containing $V_{0} a$ for some regular element $a \in A$, then

$$
\operatorname{dim}\left(W^{n}\right) \geq c \operatorname{dim}(W) n^{d}
$$

for all $n$. In this case we say $A$ satisfies $S M\left(V_{0}, c, d\right)$. A key step in proving Theorem 1.1 is to show that finitely generated commutative domains satisfy SMC [Theorem 3.2]. On the other hand, Examples 5.2 and 5.3 show that semiprime commutative algebras and noncommutative noetherian domains may not satisfy SMC.

By using Lemma 2.1(1),(4),(6) and the definition of $S M\left(V_{0}, c, d\right)$, the proof of Lemma 3.1 is straightforward and is left to the reader. Lemma 3.1 will be used in the proofs of Theorem 3.2 and Proposition 5.4.

Lemma 3.1. (1) Let $G$ be an ordered semigroup and let $A$ be a (noncommutative) $G$-filtered algebra such that the associated graded algebra $\operatorname{gr}(A)$ is a domain and that $\operatorname{GKdim}(\operatorname{gr}(A))=\operatorname{GKdim}(A)=d$. If $\operatorname{gr}(A)$ satisfies $S M\left(\nu\left(V_{0}\right), c, d\right)$, then $A$ satisfies $\operatorname{SM}\left(V_{0}, c, d\right)$.

(2) Let $A$ be a commutative domain and let $Q(A)$ be the fraction field of $A$. Then $Q(A)$ satisfies $S M\left(V_{0}, c, d\right)$ if and only if $A$ satisfies $S M\left(V_{0} y, c, d\right)$ for some $0 \neq y \in A$ such that $V_{0} y \subset A$.

Theorem 3.2. If $A$ is a commutative domain such that the fraction field $Q(A)$ is finitely generated as a field, then A satisfies SMC.

Proof. First we prove the following statement: If $R$ is a commutative domain satisfying SMC, then $R[x]$ satisfies SMC. Note that $\operatorname{GKdim}(R[x])=\operatorname{GKdim}(R)+1=$ $d+1$ [MR, 8.2.7(iii)]. Suppose now that $R$ satisfies $S M\left(V_{0}, c, d\right)$. For every subspace $W_{0} \supset V_{0} y$

$$
\operatorname{dim}\left(W_{0}^{n}\right) \geq c \operatorname{dim}\left(W_{0}\right) n^{d}
$$

for all $n$. We will verify that $R[x]$ satisfies $S M\left(V_{0}+k x, c^{\prime}, d+1\right)$ for some $c^{\prime}>0$.

Case 1. Let $W=\sum_{i=1}^{l} x^{p_{i}} W_{i}$ where the $\left\{p_{i}\right\}$ are increasing and suppose that $W_{i} \supset V_{0} y_{i}$ for some nonzero $y_{i} \in R$ for all $i$. We use induction on $l$ (for $l \geq 2$ ) to show that $\operatorname{dim}\left(W^{n}\right) \geq c_{1} \operatorname{dim}(W) n^{d+1}$. If $l=2, W=x^{p_{1}} W_{1}+x^{p_{2}} W_{2}$ and $p_{2}>p_{1}$, 
then

$$
\begin{aligned}
\operatorname{dim}\left(W^{n}\right) & \geq \operatorname{dim}\left(\sum_{s=0}^{n} x^{p_{1} s+p_{2}(n-s)} W_{1}^{s} W_{2}^{n-s}\right) \\
& =\sum_{s=0}^{n} \operatorname{dim}\left(W_{1}^{s} W_{2}^{n-s}\right)=\frac{1}{2} \sum_{s=0}^{n}\left[\operatorname{dim}\left(W_{1}^{s} W_{2}^{n-s}\right)+\operatorname{dim}\left(W_{1}^{s} W_{2}^{n-s}\right)\right] \\
& \geq \frac{1}{2} \sum_{s=0}^{n}\left(\operatorname{dim}\left(W_{1}^{s}\right)+\operatorname{dim}\left(W_{2}^{n-s}\right)\right) \\
\text { (use (SMC)) } & \geq \frac{1}{2} c\left\{\operatorname{dim}\left(W_{1}\right)+\operatorname{dim}\left(W_{2}\right)\right\} \sum_{s=0}^{n} s^{d} \\
& \geq \frac{1}{2} c\left\{\operatorname{dim}\left(W_{1}\right)+\operatorname{dim}\left(W_{2}\right)\right\} \frac{1}{2(d+1)} n^{d+1} \\
& =\frac{c}{4(d+1)} \operatorname{dim}(W) n^{d+1},
\end{aligned}
$$

where we have used the inequality $\sum_{s=0}^{n} s^{d} \geq \frac{1}{2(d+1)} n^{d+1}$ for $d \geq 0$. If $l>2$, let $W_{<l}=\sum_{i<l} W_{i} x^{p_{i}}$ and suppose now that

$$
\operatorname{dim}\left(W_{<l}^{n}\right) \geq c_{1} \operatorname{dim}\left(W_{<l}\right) n^{d+1}
$$

for all $n$ where $c_{1}=\frac{c}{4(d+1)}$. Hence we have

$$
\begin{aligned}
\operatorname{dim}\left(W^{n}\right) & \geq \operatorname{dim}\left(W_{<l}^{n} \oplus \sum_{t=1}^{n}\left(x^{p_{l-1}} W_{l-1}\right)^{n-t}\left(x^{p_{l}} W_{l}\right)^{t}\right) \\
& \geq \operatorname{dim}\left(W_{<l}^{n}\right)+\sum_{t=1}^{n} \operatorname{dim}\left(W_{l}^{t}\right) \\
& \geq c_{1} \operatorname{dim}\left(W_{<l}\right) n^{d+1}+c \operatorname{dim}\left(W_{l}\right) \sum_{t=1}^{n} t^{d} \\
& \geq c_{1} \operatorname{dim}\left(W_{<l}\right) n^{d+1}+\frac{c}{2(d+1)} \operatorname{dim}\left(W_{l}\right) n^{d+1} \\
& \geq c_{1} \operatorname{dim}\left(W_{<l}\right) n^{d+1}+c_{1} \operatorname{dim}\left(W_{l}\right) n^{d+1}=c_{1} \operatorname{dim}(W) n^{d+1} .
\end{aligned}
$$

Case 2. In general let $W$ be a subspace such that $W \supset\left(V_{0}+k x\right) y$ for some $y \in R[x]$. By Lemma 2.1(6), we have $\operatorname{dim}\left(W^{n}\right) \geq \operatorname{dim}\left(\nu(W)^{n}\right)$. Hence we only need to prove that $\operatorname{dim}\left(\nu(W)^{n}\right) \geq c^{\prime} \operatorname{dim}(W) n^{d+1}$ for all $n$. By Lemma 2.1(1), $\nu(W) \supset\left(V_{0}+k x\right) \nu(y)$. Let $\nu(y)=a x^{p}$. Then $\nu(W) \supset V_{0} a x^{p}+k a x^{p+1}$. Hence $W^{\prime}:=V_{0} a x^{p} \nu(W)\left(\subset \nu(W)^{2}\right)$ satisfies the conditions in case 1. By case 1,

$$
\operatorname{dim}\left(\left(W^{\prime}\right)^{n}\right) \geq c_{1} \operatorname{dim}\left(W^{\prime}\right) n^{d+1} \geq c_{1} \operatorname{dim}(\nu(W)) n^{d+1}=c_{1} \operatorname{dim}(W) n^{d+1} .
$$

Hence

$$
\begin{aligned}
\operatorname{dim}\left(W^{n}\right) & \geq \operatorname{dim}\left(\left(\nu(W)^{2}\right)^{\left[\frac{n}{2}\right]}\right) \geq \operatorname{dim}\left(W^{\prime\left[\frac{n}{2}\right]}\right) \\
& \geq c_{1} \operatorname{dim}(W)\left[\frac{n}{2}\right]^{d+1} \geq \frac{c_{1}}{2^{d+2}} \operatorname{dim}(W) n^{d+1} .
\end{aligned}
$$

Therefore $R[x]$ satisfies $S\left(V_{0}+k x, c^{\prime}, d+1\right)$ where $c^{\prime}=\frac{c_{1}}{2^{d+2}}$ and the statement is proved. 
It is trivial to check that $k$ satisfies $\operatorname{SMC}$ with $d=\operatorname{GKdim}(k)=0$. By induction and the statement we just proved, the polynomial algebra $k\left[x_{1}, \cdots, x_{d}\right]$ satisfies $S M\left(V_{0}, c, d\right)$ for $V_{0}=k+\sum_{i=1}^{d} k x_{i}$.

Now we prove that every commutative domain $A$ with $Q(A)$ being finitely generated satisfies SMC.

By using Lemma 3.1(2) twice, we may assume that $A$ is a finitely generated commutative domain. Consider the variety $Y$ defined by the algebra $A$. By $[\mathrm{Ha}$, I.5.3], there is a nonsingular point $p \in Y$. By [Ha, I.5.1], the local ring at the point $p, B:=\mathcal{O}_{p, Y}$, is regular. Hence $Q(B)=Q(A)$ and the Krull dimension of $B$ is equal to the Krull dimension of $A$, which is also equal to the GK dimension of $A$ [KL, 4.5]. By Lemma 3.1(2) again, we may assume that $A$ is a regular local algebra of Krull dimension and GK dimension $d$. By [Ma, 14.4], $A$ is a $\mathbb{Z}$-filtered algebra with associated graded algebra $\operatorname{gr}(A)$ isomorphic to the polynomial ring $k\left[x_{1}, \cdots, x_{d}\right]$. Since the polynomial algebra satisfies SMC, by Lemma 3.1(1), $A$ satisfies SMC and thus we have finished our proof.

In the proof of Theorem 3.2, we used the fact that $k$ is algebraically closed. It is unknown if a (finitely generated) commutative domain over an arbitrary field satisfies SMC.

Proposition 3.3. Let $A$ be an algebra and let $\sigma$ be an algebra endomorphism of A.

(1) If $A$ is a commutative algebra satisfying SMC and if $\sigma$ is not locally algebraic, then $\operatorname{GKdim}(A[x, \sigma]) \geq \operatorname{GKdim}(A)+2$.

(2) If $\sigma$ is locally algebraic, then $\operatorname{GKdim}(A[x, \sigma])=\operatorname{GKdim}(A)+1$.

Proof. By definition $V x=x V^{\sigma}$ where $V^{\sigma}=\sigma(V)$. For every subspace $V$ of $A$, we have

$$
\begin{aligned}
(V+k x)^{n} & =\sum_{i=0}^{n}\left(\sum_{\sum k_{s}=n-i} V^{k_{i}} x V^{k_{i-1}} x \cdots x V^{k_{0}}\right) \\
& =\sum_{i=0}^{n} \sum_{\sum k_{s}=n-i} x^{i}\left(V^{\sigma^{i}}\right)^{k_{i}}\left(V^{\sigma^{i-1}}\right)^{k_{i-1}} \cdots(V)^{k_{0}} \\
& =\sum_{i=0}^{n} x^{i} W_{n, i},
\end{aligned}
$$

where $W_{n, i}=\sum_{\sum k_{s}=n-i}\left(V^{\sigma^{i}}\right)^{k_{i}}\left(V^{\sigma^{i-1}}\right)^{k_{i-1}} \cdots(V)^{k_{0}}$.

(1) If $A$ is commutative, we have

$$
W_{n, i}=\sum_{\sum k_{s}=n-i}\left(V^{\sigma^{i}}\right)^{k_{i}}\left(V^{\sigma^{i-1}}\right)^{k_{i-1}} \cdots(V)^{k_{0}}=\left(V+V^{\sigma}+\cdots+V^{\sigma^{i}}\right)^{n-i} .
$$

If $\sigma$ is not locally algebraic, then there exists a $y \in A$ such that the subspace $\sum_{s \geq 0} k \sigma^{s}(y)$ is infinite dimensional. Let $V=V_{0}+k y$ for some $V_{0}$ and let $W_{i}=$ $V+V^{\sigma}+\cdots+V^{\sigma^{i}}$. Then $\operatorname{dim}\left(W_{i}\right) \geq \operatorname{dim}\left(\sum_{s=0}^{i} k \sigma^{s}(y)\right)=i+1$ for all $i$. By (3.3.1) and (3.3.2), we have

$$
(V+k x)^{n}=\sum_{i=0}^{n} x^{i} W_{n, i}=\sum_{i=0}^{n} x^{i}\left(W_{i}\right)^{n-i} .
$$


Since $A$ satisfies SMC, say $S M\left(V_{0}, c, d\right), \operatorname{dim}\left(W_{i}^{n-i}\right) \geq c \operatorname{dim}\left(W_{i}\right)(n-i)^{d}$. Hence

$$
\begin{aligned}
\operatorname{dim}(V+k x)^{n} & =\sum_{i=0}^{n} \operatorname{dim}\left(W_{i}^{n-i}\right) \geq \sum_{i=0}^{n} c \operatorname{dim}\left(W_{i}\right)(n-i)^{d} \\
& \geq \sum_{i=0}^{n} c i(n-i)^{d} \geq \frac{c}{4^{d+2}} n^{d+2} .
\end{aligned}
$$

Therefore $\operatorname{GKdim}(A[x, \sigma]) \geq \operatorname{GKdim}(A)+2$.

(2) If $\sigma$ is locally algebraic and $V$ is $\sigma$-stable, by (3.3.1), we have $\operatorname{dim}\left((V+k x)^{n}\right)=$ $\sum_{i=0}^{n} \operatorname{dim}\left(V^{n-i}\right)$. This implies that $\operatorname{GKdim}(A[x, \sigma])=\operatorname{GKdim}(A)+1$.

In general the GKdim of $A[x, \sigma]$ could jump by more than 2. By [MR, page 291] there is an automorphism $\sigma$ of $A=k\left[y^{ \pm 1}, z^{ \pm 1}\right] \operatorname{such}$ that $\operatorname{GKdim}(A[x, \sigma])=$ $\operatorname{GKdim}\left(A\left[x^{ \pm 1}, \sigma\right]\right)=\infty$. Here $\left\{x^{ \pm 1}\right\}$ means $\left\{x, x^{-1}\right\}$ (and similarly for other variables).

Proof of Theorem 1.1. By Proposition 3.3(2) (c) implies (b), and by [LMO, Prop.1] (c) implies (b) ${ }^{\prime}$. It is trivial that (b) implies (a), (b)' implies (a)', and (a)' implies (a). Hence it remains to prove that (a) implies (c). But this implication is an immediate consequence of Theorem 3.2 and Proposition 3.3(1).

Next we assume that $A$ is a finitely generated field and show that (c) and (d) are equivalent. It is easy to see that (d) implies (c). Hence it remains to verify that (c) implies (d). Now suppose that $\sigma$ is a locally algebraic endomorphism of $A$. Let $V$ be a $\sigma$-stable subframe which generates $A$ as a field. Consider $\sigma$ as a $k$-linear map from $V$ to itself. Since $k$ is algebraically closed, there are $l$ eigenvalues of $\sigma$ where $l=\operatorname{dim}(V)$, and for an eigenvalue $r$, there is an eigenvector $v \in V$ such that $\sigma(v)=r v$. Since $A$ is a field, $\sigma$ is injective and hence $r$ is not zero. If $r$ is not a root of 1 , then $v \notin k$ and $\sum_{s \geq 0} k \sigma^{s}\left(\frac{1}{1-v}\right)$ is infinite dimensional. This contradicts the fact that $\sigma$ is locally algebraic. Hence every eigenvalue of $\sigma$ is a root of 1 . Replacing $\sigma$ by $\sigma^{m}$ for some positive integer $m$, we may assume that every eigenvalue of $\sigma$ is 1. In this case, $(\sigma-i d)^{n}=0$ for $n \geq l$, where $i d$ is the identity map of $V$. If the characteristic of $k$ is $p>0$, then

$$
\sigma^{p^{s}}=(i d+(\sigma-i d))^{p^{s}}=i d^{p^{s}}+(\sigma-i d)^{p^{s}}=i d
$$

for $s \gg 0$. If the characteristic of $k$ is 0 , then $\sigma$ is the identity. If not, then there is a $v \in V$ such that $(\sigma-i d)^{t+1}(v)=0$ and $(\sigma-i d)^{t}(v) \neq 0$ for some $t>0$. Let $x=(\sigma-i d)^{t}(v)$ and $y=(\sigma-i d)^{t-1}(v)$. Hence $\sigma(x)=x$ and $\sigma(y)=y+x$. Thus $\sum_{i} k \sigma^{i}\left(\frac{x}{y}\right)$ is infinite dimensional, a contradiction. Therefore, for any characteristic, $\sigma^{n}=i d$ as a $k$-linear endomorphism of $V$ for some $n>0$. Since $V$ generates the field $A, \sigma$ is an automorphism of $A$ of finite order. Thus we have finished our proof.

\section{Proof of Theorem 1.2}

To prove Theorem 1.2 we need the following lemma which is an analogue of [KL, $3.5]$.

Lemma 4.1. Let $A$ be an algebra, $\sigma$ an endomorphism of $A$, and $\delta$ a $\sigma$-derivation of $A$. If every subframe $W$ of $A$ is contained in a $\sigma$-stable subframe $V$ such that the subalgebra $k[V]$ is $\delta$-stable, then $\operatorname{GKdim}(A[x, \sigma, \delta])=\operatorname{GKdim}(A)+1$. 
Proof. We copy the proof of $[\mathrm{KL}, 3.5]$ with a slight modification. Every subframe of $A[x, \sigma, \delta]$ is contained in $(V+k x)^{s}$ for some subframe $V$ of $A$ and some integer $s$. Hence to compute $\operatorname{GKdim}(A[x, \sigma, \delta])$ we only need to consider subframes $V+k x$. By the hypothesis, we may assume $V$ is $\sigma$-stable and the subalgebra $k[V]$ is $\delta$-stable. Hence $\delta(V) \subset V^{m}$ for some fixed integer $m$. By induction on $l$ and by the fact that $V$ is $\sigma$-stable it is easy to prove that

$$
\delta\left(V^{l}\right) \subset V^{l+m-1}
$$

for all $l \geq 1$. By induction on $n$, we have

$$
(V+k x)^{n} \subset V^{n m}+x V^{n m}+\cdots+x^{n} V^{n m} .
$$

This implies that $\operatorname{GKdim}(A[x, \sigma, \delta]) \leq \operatorname{GKdim}(A)+1$. Since $A[x, \sigma, \delta]$ is a filtered algebra with associated graded algebra $A[x, \sigma]$, by Proposition 3.3(2) and Lemma $2.1(7)$ we have $\operatorname{GKdim}(A[x, \sigma, \delta]) \geq \operatorname{GKdim}(A)+1$. Therefore $\operatorname{GKdim}(A[x, \sigma, \delta])=$ $\operatorname{GKdim}(A)+1$.

Proof of Theorem 1.2. (1) It is trivial that (b) implies (a).

(a) $\Rightarrow$ (c) Note that $A[x, \sigma, \delta]$ is a filtered algebra with associated graded algebra $A[x, \sigma]$. By Lemma 2.1(7), $\operatorname{GKdim}(A[x, \sigma]) \leq \operatorname{GKdim}(A[x, \sigma, \delta])<\operatorname{GKdim}(A)+2$. By Theorem 1.1, $\sigma$ is locally algebraic.

(c) $\Rightarrow$ (b) If $A$ is finitely generated and $\sigma$ is locally algebraic, then the hypothesis of Lemma 4.1 holds. By Lemma 4.1, $\operatorname{GKdim}(A[x, \sigma, \delta])=\operatorname{GKdim}(A)+1$.

(2) It is trivial that (b) implies (a). As in the proof of (1), (a) implies (c). By the proof of Theorem 1.1, (c) is equivalent to (d). It remains to prove that (d) implies (b). Suppose now that $\sigma$ is of finite order. Since $A$ is finitely generated, every subframe of $A$ is contained in a $\sigma$-stable subframe $V=\sum_{j=1}^{n} k x_{j}$ of $A$ which generates $A$ as a field. For every $j$, there exist $f_{j}, g_{j} \in k[V]$ such that $\delta\left(x_{j}\right)=f_{j} g_{j}^{-1}$. Let $g$ be the product $\prod_{i=0}^{q-1} \prod_{j=1}^{n} \sigma^{i}\left(g_{j}\right)$ where $q$ is the order of $\sigma$. Then $\sigma(g)=g$ and $\delta\left(x_{j}\right)=f_{j}^{\prime} g^{-1}$ for some $f_{j}^{\prime} \in k[V]$. Hence $W=V+k g^{-1}$ is a $\sigma$-stable subframe of $A$. We claim that $k[W]$ is $\delta$-stable. Since $W$ is $\sigma$-stable, it suffices to prove that $\delta(W) \subset k[W]$. By the definition of $g, \delta(V) \subset k[W]$. Since $V$ is $\sigma$-stable, $\delta(k[V]) \subset k[W]$. In particular, $\delta(g) \in k[W]$. It is easy to see that $\delta\left(g^{-1}\right)=-g^{-2} \delta(g) \in k[W]$. Therefore $\delta(W) \subset k[W]$ and the hypothesis of Lemma 4.1 holds. By Lemma 4.1, $\operatorname{GKdim}(A[x, \sigma, \delta])=\operatorname{GKdim}(A)+1$.

\section{EXAMPLES}

In this section we will see that not every algebra satisfies SMC. To measure in what degree an algebra satisfies SMC, we define the following invariant. Given a subspace $V_{0}$ and positive constants $c$ and $d$, we say $A$ satisfies $S M\left(V_{0}, c, d\right)$ if for every subspace $W \supset V_{0} a$ for some regular element $a \in A$,

$$
\operatorname{dim}\left(W^{n}\right) \geq c \operatorname{dim}(W) n^{d}
$$

for all $n \geq 1$. The growth of $A$ with sensitive multiplicity (or SM-growth of $A$ ) is defined to be

$$
g(A)=\sup \left\{d \mid A \text { satisfies } S M\left(V_{0}, c, d\right) \text { for some } V_{0} \text { and } c\right\} .
$$

By definition, $g(A) \leq \mathrm{GKdim}(A)$. If $A$ satisfies SMC, then $g(A)=\operatorname{GKdim}(A)$. In other words, if $g(A)<\operatorname{GKdim}(A)$, then $A$ does not satisfy SMC. 
Next we will give some examples in which $g(A)<\operatorname{GKdim}(A)$, and show that (a) and (c) in Theorem 1.1 are not equivalent for these algebras. Most of the following statements are easily checked, or follow from direct computations, thus detailed proofs will be omitted.

Example 5.1. Let $A$ be the finitely generated commutative algebra $k\left[x^{ \pm 1}, y\right] /\left(y^{2}\right)$. Then $\operatorname{GKdim}(A)=1$ and $g(A)=0$. Let $\sigma$ be the automorphism of $A$ defined by $\sigma: x \longmapsto x, x^{-1} \longmapsto x^{-1}$, and $y \longmapsto x y$. The subspace $\sum_{s>0} k \sigma^{s}(y)$ is infinite dimensional and hence $\sigma$ is not locally algebraic. By a direct computation, we have

$$
\operatorname{GKdim}\left(A\left[z^{ \pm 1}, \sigma\right]\right)=\operatorname{GKdim}(A)+1=2 .
$$

Therefore (a) and (c) in Theorem 1.1 are not equivalent for this commutative algebra.

Example 5.2. Let $B$ be the field $k(t)$ and let $\alpha$ be the automorphism of $B$ defined by $\alpha(t)=q t$ for some nonzero scalar $q$. If $q$ is not a root of 1 , then $\alpha$ is not of finite order and $\operatorname{GKdim}(B[z, \alpha])=3>\operatorname{GKdim}(B)+1$. However, $g(B[z, \alpha])=2=$ $g(B)+1$.

Let $A$ be the commutative algebra $k[x, y] \oplus k(t)$. Then $A$ is a semiprime algebra but not a domain. It is easy to see that $g(A)=g(k(t))=1$ and $\operatorname{GKdim}(A)=$ $\operatorname{GKdim}(k[x, y])=2$. Let $\sigma$ be the automorphism defined by $\left.\sigma\right|_{k[x, y]}=i d$ and $\left.\sigma\right|_{k(t)}=\alpha$. Then $\operatorname{GKdim}\left(A\left[z^{ \pm 1}, \sigma\right]\right)=3$ but $\sigma$ is not locally algebraic. Hence (a) and (c) in Theorem 1.1 are not equivalent for the algebra $A$.

Example 5.3. Let $G$ be the nilpotent group $\langle y, z|[y, z]=x$ central $\rangle$. Then the group algebra $k[G]$ is isomorphic to $k\left[x^{ \pm 1}, y^{ \pm 1}\right]\left[z^{ \pm 1}, \sigma\right]$ where $\sigma$ is defined by $\sigma: x \longmapsto x, y \longmapsto x y$. By $[\mathrm{KL}, 11.10], \operatorname{GKdim}(k[G])=4$. To prove $g(k[G])<$ $\operatorname{GKdim}(k[G])$ we consider the subspace

$$
V=\sum k x^{i} y^{j} z^{s} \quad \text { where } \quad|i| \leq m,|j| \leq l,|s| \leq l .
$$

By direct computation (and of course using $\sigma$ ), we see that

$$
\begin{aligned}
V^{n} \subset W_{n}:=\sum k x^{i} y^{j} z^{s} \\
\quad \text { where }|i| \leq m \cdot n+\frac{1}{2} n(n+1) l^{2},|j| \leq l \cdot n,|s| \leq l \cdot n .
\end{aligned}
$$

It is obvious that

$$
\operatorname{dim}(V)=(2 m+1)(2 l+1)^{2}
$$

and

$$
\operatorname{dim}\left(W_{n}\right)=\left(2 m n+n(n+1) l^{2}\right)(2 l n+1)^{2} .
$$

If we let $m$ go to infinity, $\operatorname{dim}\left(W_{n}\right) \approx 8 m l^{2} n^{3}$ and $\operatorname{dim}(V) \approx 8 m l^{2}$. Then $\operatorname{dim}\left(V^{n}\right) \leq$ $\operatorname{dim}\left(W_{n}\right)$ implies that $g(k[G]) \leq 3$. Since $x-1$ is a central regular element of $k[G]$ and $k[G] /(x-1) \cong k\left[y^{ \pm 1}, z^{ \pm 1}\right], k[G]$ is a $\mathbb{Z}$-filtered algebra with associated graded algebra isomorphic to $k\left[y^{ \pm 1}, z^{ \pm 1}\right][X][\mathrm{Zh}, 6.3]$. By using the leading-term map, we can prove that $g(k[G]) \geq g\left(k\left[y^{ \pm 1}, z^{ \pm 1}\right][X]\right)=3$. Thus we conclude that $g(k[G])=3<\operatorname{GKdim}(k[G])=4$.

To see that (a) and (c) in Theorem 1.1 are not equivalent for $A=k[G]$ we consider the automorphism $\delta$ of $A$ defined by $\delta: x \longmapsto x, y \longmapsto y$ and $z \longmapsto x^{-1} z$. Hence $\sum_{s \geq 0} k \delta^{s}(z)$ is infinite dimensional and this implies that $\delta$ is not locally algebraic. It remains to prove that $\operatorname{GKdim}\left(A\left[t^{ \pm 1}, \delta\right]\right)=\operatorname{GKdim}(A)+1$. We rewrite 
$A\left[t^{ \pm 1}, \delta\right]$ as $k\left[x^{ \pm 1}, y^{ \pm 1}, t^{ \pm 1}\right]\left[z^{ \pm 1}, \alpha\right]$ where $\alpha$ is the algebra automorphism defined by $\alpha: x \longmapsto x, y \longmapsto x y, t \longmapsto x t$. Using formulas (3.3.1) and (3.3.2), we can check directly that $\operatorname{GKdim}\left(k\left[x^{ \pm 1}, y^{ \pm 1}, t^{ \pm 1}\right]\left[z^{ \pm 1}, \alpha\right]\right)=5$. Therefore $\operatorname{GKdim}\left(A\left[t^{ \pm 1}, \delta\right]\right)=$ $\operatorname{GK} \operatorname{dim}(A)+1$ but $\delta$ is not locally algebraic. Finally it can also be verified that $g\left(k[G]\left[t^{ \pm 1}, \delta\right]\right)=4=g(k[G])+1$.

Though we can not expect Theorems 1.1, 1.2 and 3.2 hold for an arbitrary noncommutative domain, we can still try to prove a similar result for some special noncommutative domains. Here we prove that Theorem 3.2 holds for some nice noncommutative domains. It will be interesting to see if Theorems 1.1 and 1.2 hold for the domains listed next.

Proposition 5.4. The following (noncommutative) domains satisfy SMC.

(1) Skew polynomial algebras $k_{p_{i j}}\left[x_{1}, \cdots, x_{d}\right]$ and $k_{p_{i j}}\left[x_{1}^{ \pm 1}, \cdots, x_{d}^{ \pm 1}\right]$.

(2) The Weyl algebras $A_{d}$.

(3) The universal enveloping algebras $U(L)$ of finite dimensional Lie algebras $L$.

(4) Quantum matrix algebras $M_{d}\left(q, p_{i j}\right)$ and $G L_{d}\left(q, p_{i j}\right)$.

(5) Quantum Weyl algebras $A_{d}\left(q, p_{i j}\right)$.

Proof. (1) It is easy to see that $k_{p_{i j}}\left[x_{1}, \cdots, x_{d}\right]$ is a twisted semigroup algebra $k * \mathbb{N}^{d}$ and that $k_{p_{i j}}\left[x_{1}^{ \pm 1}, \cdots, x_{d}^{ \pm 1}\right]$ is a twisted group algebra $k * \mathbb{Z}^{d}$. By Theorem 3.2, the commutative polynomial algebras $k\left[\mathbb{N}^{d}\right]$ and $k\left[\mathbb{Z}^{d}\right]$ satisfy SMC. Hence it suffices to prove the following statement: If $G$ is an ordered semigroup, then a twisted semigroup algebra $k * G$ [MR, 1.5.8] satisfies SMC if and only if the semigroup algebra $k[G]$ satisfies $\operatorname{SMC}$. Note that $\operatorname{GKdim}(k * G)=\operatorname{GKdim}(k[G])[\mathrm{Zh}, 8.5(1)]$.

By definition, $k * G$ and $k[G]$ have the same $G$-graded $k$-linear space $k G$. Since $k * G$ is a $G$-graded algebra and $G$ is ordered, there is a leading-term map $\nu$ from $k * G$ to itself. Hence we can apply Lemma 2.1 to the graded algebra $k * G$. As in the proof of Theorem 3.2 (see case 2), we only need to consider $G$-graded subspaces to prove $S M\left(V_{0}, c, d\right)$ for some graded subspace $V_{0}$. For every $G$-graded subspace $W$ of $k * G, W=k H$ for some subset $H \subset G$. Let $V$ be another $G$-graded subspace $k L$ for some $L \subset G$. Then it is easy to check that

$$
W V=k(H L) \quad \text { and } \quad W^{n}=k\left(H^{n}\right)
$$

as subspaces of $k * G$. Hence the dimensions of the subspaces $W, V, W V$ and $W^{n}$ are only dependent on the subsets of the semigroup $G$ and the group multiplication. By the definition of $S M\left(V_{0}, c, d\right), k * G$ satisfies $S M\left(V_{0}, c, d\right)$ if and only if $k[G]$ does.

(2)-(5) By Lemma 3.1(1) and part (1), it is enough to check that every algebra in $(2)-(5)$ is a $\mathbb{Z}^{l}$-filtered algebra such that the associated graded algebra $\operatorname{gr}(A)$ is a commutative or skew polynomial algebra and that $\operatorname{GKdim}(A)=\operatorname{GKdim}(\operatorname{gr}(A))$.

By [Zh, 6.9], the Weyl algebras and universal enveloping algebras are $\mathbb{Z}$-filtered algebras with associated graded algebras being commutative polynomial rings. By [Zh, 7.4], the quantum algebra $M_{d}\left(q, p_{i j}\right)$ is a $\mathbb{Z}^{d^{2}}$-filtered algebra with associated graded algebra being $k * \mathbb{N}^{d^{2}}$. By [Zh, 7.4], the quantum algebra $G L_{d}\left(q, p_{i j}\right)$ is a $\mathbb{Z}^{d^{2}}$-filtered algebra with associated graded algebra being $k *\left(\mathbb{N}^{d^{2}-d} \oplus \mathbb{Z}^{d}\right)$. By [Zh, 7.5], the $A_{d}\left(q, p_{i j}\right)$ is a $\mathbb{Z}^{2 d}$-filtered algebra with associated graded algebra being $k * \mathbb{N}^{2 d}$. Finally $[\mathrm{Zh}, 6.9,7.4$ and 7.5] also indicate that $\operatorname{GKdim}(A)=\operatorname{GKdim}(\operatorname{gr}(A))$ for the algebras in (2)-(5) and thus we have finished our proof. 


\section{ACKNOWLEDGMENT}

The author would like to thank S. Paul Smith and Ron Irving for useful conversations and helpful comments on the subject.

\section{REFERENCES}

[Ha] R. Hartshorne, Algebraic Geometry, Springer-Verlag, New York, 1977. MR 57:3116

[KL] G. Krause and T. H. Lenagan, Growth of algebras and Gelfand-Kirillov dimension, Research Notes in Mathematics, Pitman Adv. Publ. Program 116 (1985). MR 86g:16001

[LMO] A. Leroy, J. Matczuk and J. Okninski, On the Gelfand-Kirillov dimension of normal localizations and twisted polynomial rings, Perspectives in Ring Theory (F. van Oystaeyen and L. Le Bruyn, eds.), Kluwer Academic Publishers, 1988, pp. 205-214. MR 91c:16020

[Ma] H. Matsumura, Commutative Ring Theory, (Translated by M. Reid), Cambridge University Press, 1986. MR 88h:13001

[MR] J. C. McConnell and J. C. Robson, Non-Commutative Noetherian Rings, Wileyinterscience, Chichester, 1987. MR 89j:16023

[Mu I. Musson, Gelfand-Kirillov dimension of twisted Laurent extensions, Comm. Alg., vol. 17 (11), 1989, pp. 2853-2856. MR 91a:16018

[Zh] J. J. Zhang, On Gelfand-Kirillov transcendence degree, Trans. Amer. Math. Soc. 348 (1996), 2867-2899.

Department of Mathematics, Box 354350, University of Washington, Seattle, WashINGTON 98195

E-mail address: zhang@math.washington.edu 